\title{
A Linear Programming Approach to the Development of Contrail Reduction Strategies Satisfying Operationally Feasible Constraints
}

\author{
Peng Wei * Banavar Sridhar ${ }^{\dagger}$ Neil Chen ${ }^{\dagger}$ Dengfeng Sun*
}

\begin{abstract}
A class of strategies has been proposed to reduce contrail formation in the United States airspace. A 3D grid based on weather data is built and the cruising altitude level of aircraft is adjusted to avoid the persistent contrail potential area with the consideration to fuel-efficiency. In this paper, the authors introduce a contrail avoidance strategy on 3D grid by considering additional operationally feasible constraints from an air traffic controllers aspect. First, shifting too many aircraft to the same cruising level will make the miles-in-trail at this level smaller than the safety separation threshold. Furthermore, the high density of aircraft at one cruising level may exceed the manageable workload for the traffic controller. Therefore, in our new model we restrict the number of total aircraft at each level. Second, the aircraft count variation for successive intervals can not be too drastic since the workload to manage climbing/descending aircraft is much larger than managing cruising aircraft. The contrail reduction problem is formulated as integer programming and the problem is shown to have the property of total unimodularity. Solving the corresponding relaxed linear programming with the simplex method provides an optimal and integral solution to the problem. Simulation results are provided to illustrate the methodology.
\end{abstract}

\section{Nomenclature}

$T$ temperature in Celsius

$R H w$ relative humidity with respect to water

$R H i$ relative humidity with respect to ice

$l \quad$ cruising altitude level index

$R_{t}^{l} \quad$ potential persistent contrail formation matrix (contrail matrix) at time $t$ at level $l$

$A_{t}^{l} \quad$ aircraft position matrix at time $t$ at level $l$

CFI center contrail frequency index

$\Delta l_{f e}$ level changing constraint

$b_{1}(l)$ the number of aircraft which cruise at level $l$ in the original flight plans

$b_{2}(l)$ center level capacity at level $l$

$\Delta q$ given threshold of climbing/descending aircraft count

\section{Introduction}

Contrails are artificial clouds that are the visible trails of condensed water vapor made by the exhaust of aircraft engines. Depending on atmospheric conditions, contrails may be visible for only a few seconds or minutes, or may persist for many hours which may affect climate. Persistent contrails reduce incoming solar radiation and outgoing thermal radiation in a way that accumulates heat. ${ }^{1}$ The global mean contrail cover in 1992 was estimated to double by 2015 , and quadruple by 2050 due to air traffic increase. ${ }^{2}$ Studies suggest that the environmental impact from persistent contrail is estimated to be three or four times, ${ }^{3}$ or even ten

*School of Aeronautics and Astronautics, Purdue University

${ }^{\dagger}$ NASA Ames Research Center, Moffett Field, CA 
times ${ }^{4}$ larger than aviation-induced emissions. Therefore, strategies and policies to reduce aircraft induced persistent contrail need to be studied to minimize the impact on the global environment.

There have been some research works accomplished to identify and reduce persistent contrail formation. Gierens ${ }^{5}$ and Noppel $^{6}$ reviewed various strategies for contrail avoidance including changing engine architecture, enhancing airframe and engine integration, using alternate fuels, and modifying traffic flow management procedures. Among the traffic flow management solutions, Mannstein ${ }^{7}$ presented a strategy to reduce the environment impact of contrails significantly by small changes to each aircraft's flight altitude. Campbell ${ }^{8}$ proposed a mixed integer programming method to optimally reroute aircraft to avoid the formation of persistent contrails. Both methods require the onboard contrail detection system. Fichter ${ }^{9}$ showed that the global annual mean contrail coverage could be reduced by decreasing the aircraft cruise altitude. Williams ${ }^{10,11}$ proposed strategies for contrail reduction by identifying fixed and varying maximum altitude restrictions. These restrictions generally require more fuel burn and add congestion to the already crowded airspace at lower altitudes. Sridhar et al. ${ }^{12}$ provided a set of strategies to reduce contrail formation. A 3D grid model was constructed based on weather data to describe the contrail potential areas and the cruising level of aircraft is adjusted to avoid these areas with the consideration of fuel-efficiency.

The goal of this paper is to refine the research work in ${ }^{12}$ by introducing two operationally feasible constraints from air traffic control perspective with the consultation from experienced and expert controllers. Moreover, we formulate the problem as integer programming. Because of the total unimodularity of its constraint matrix, solving the relaxed linear programming by simplex method will yield the optimal and integral solution with much shorter computation time.

The rest of the paper is organized as follows. Section II introduces the atmospheric data, contrail formation model, aircraft data and contrail frequency index used in this paper. Section III presents the problem formulation and our contrail reduction strategy. The numerical results are shown in Section IV. Section V summarizes and concludes the paper.

\section{Data and Model}

\section{A. Atmospheric Data}
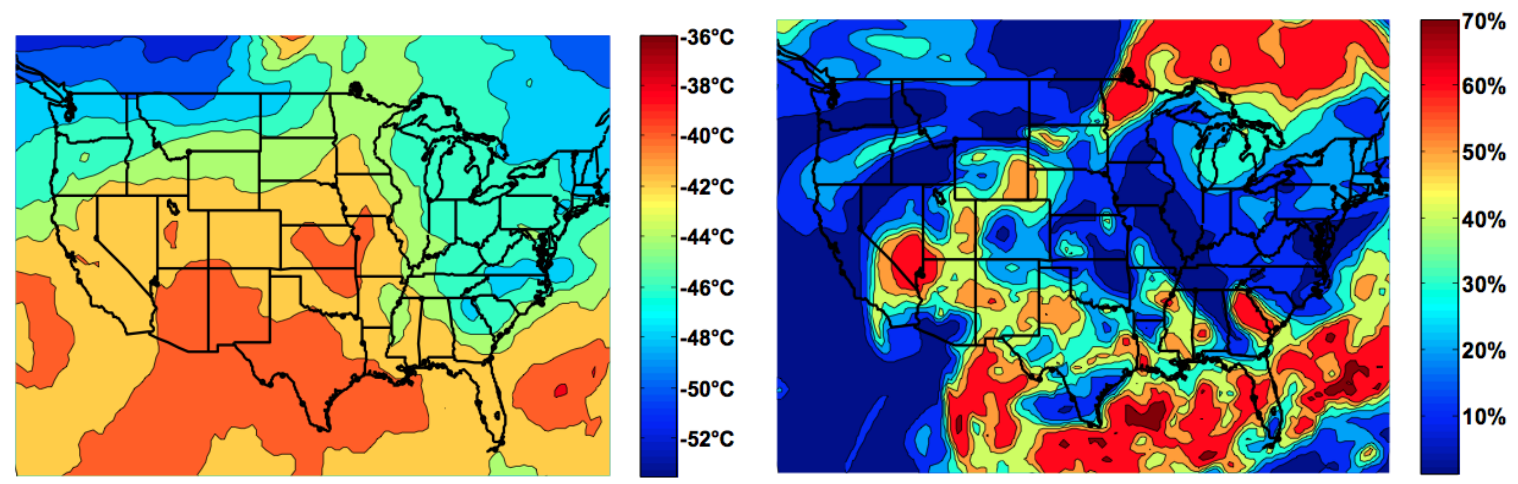

Figure 1. Contours of temperature and RHw at 34,057 feet at 8AM EDT on August 1, 2007.

Contrails can be observed from surface data ${ }^{13}$ and detected by satellite data. ${ }^{14}$ Duda $^{15}$ has related the observations to numerical weather analysis output and showed that persistent contrail formation can be computed using atmospheric temperature and humidity data retrieved from the Rapid Updated Cycle (RUC) data provided by the National Oceanic and Atmospheric Administration (NOAA). Contrails can persist when ambient air is supersaturated with respect to ice, which means that relative humidity with respect to ice (RHi) is greater than one hundred percent. ${ }^{16}$ The RHi can be computed from relative humidity with respect to water $(\mathrm{RHw})$ and temperature, which are available in the RUC data. The one-hour forecast and the 40-km RUC data are used in this paper. The data have a temporal resolution of one hour, a horizontal resolution of 40 kilometers, and isobaric pressure levels from 100 to 1000 hectopascals (hPa) with $25 \mathrm{hPa}$ increments. The vertical range of interest in this study is from $150 \mathrm{hPa}$ to $400 \mathrm{hPa}$, which is equivalent to pressure altitude of about 23,600 feet to 44,400 feet. The temperature and RHw contours at 8AM eastern 
daylight time (EDT) on August 1, 2007 at pressure altitude $250 \mathrm{hPa}$, or 34,057 feet, are shown as the left and right subfigures in Fig. 1.

\section{B. Contrail Formation Model}

The potential persistent contrail formation areas (contrail areas) are defined as areas with RHi greater than or equal to $100 \%$. RHi can be computed from RHw and temperature using the saturation vapor pressure coefficients of Alduchov, ${ }^{17}$ formulated as

$$
R H i=R H w \times \frac{6.0612 e^{18.102 T /(249.52+T)}}{6.1162 e^{22.577 T /(237.78+T)}},
$$

where $T$ is the temperature in Celsius. The atmospheric profile shown in the left and right subfigures in Fig. 1 can be translated to a contour of RHi, as shown in Fig. 2.

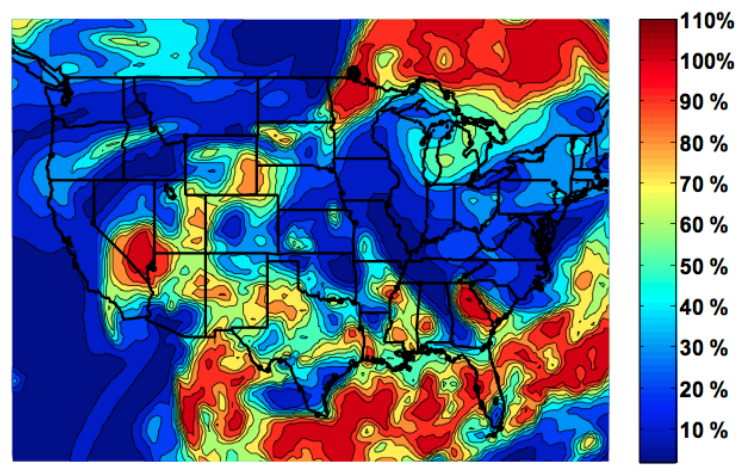

Figure 2. Contour of RHi at 34,057 feet at 8AM EDT on August 1, 2007.

The $40-\mathrm{km}$ RUC data have $(113 \times 151)$ data points. The altitude level index $l$ is defined as $l=1,2, \ldots, 11$ corresponding to isobaric pressure level at 400,375, .., $150 \mathrm{hPa}$. The level index, isobaric pressure level, and approximate aircraft cruising altitude are listed in Table 1.

Table 1. Level index, isobaric pressure level and approximate aircraft cruising altitude.

\begin{tabular}{cccccccccccc}
\hline \hline Level index & 1 & 2 & 3 & 4 & 5 & 6 & 7 & 8 & 9 & 10 & 11 \\
\hline Pressure level (hPa) & 400 & 375 & 350 & 325 & 300 & 275 & 250 & 225 & 200 & 175 & 150 \\
\hline Cruising altitude (100 feet) & 236 & 251 & 267 & 283 & 301 & 320 & 341 & 363 & 387 & 414 & 444 \\
\hline \hline
\end{tabular}

The potential persistent contrail formation matrix (contrail matrix) at time $t$ at level $l$ is defined as

$$
R_{t}^{l}=\left(\begin{array}{cccc}
r_{1,1} & r_{1,2} & \ldots & r_{1,151} \\
\vdots & \vdots & \ddots & \vdots \\
r_{113,1} & r_{113,2} & \ldots & r_{113,151}
\end{array}\right)
$$

where $r_{i, j}$ is 1 if $\mathrm{RHi} \geq 100 \%$ at grid $(i, j)$ and 0 if $\mathrm{RHi}<100 \%$.

\section{Aircraft Data}

Contrails form when aircraft fly through a potential contrail formation area. Thus aircraft locations are needed to determine the contrail formation frequency. The aircraft data used in this paper are obtained from the aircraft locations provided by the Federal Aviation Administration's (FAA's) Aircraft Situation Display to Industry (ASDI) data. The ASDI has a sampling rate of one minute. The same 3D grid used in 
the RUC data is used to generate the aircraft position matrix. The aircraft position matrix is defined as

$$
A_{t}^{l}=\left(\begin{array}{cccc}
a_{1,1} & a_{1,2} & \ldots & a_{1,151} \\
\vdots & \vdots & \ddots & \vdots \\
a_{113,1} & a_{113,2} & \ldots & a_{113,151}
\end{array}\right)
$$

where $a_{i, j}$ is the number of aircraft within grid $(i, j)$ flying closest to level $l$ at time $t$. The aircraft position matrix indicates the air traffic density in the grid scale at different altitudes.

\section{Contrail Frequency Index}

Contrail frequency index is defined as the number of aircraft that would fly through potential contrail formation regions during a period of time. Center contrail frequency index is used to indicate the contrail severity in a given center. ${ }^{18}$ To specify the location of the twenty U.S. centers in the grid scale, the center grid matrix is defined as

$$
C_{\text {center }}=\left(\begin{array}{cccc}
c_{1,1} & c_{1,2} & \ldots & c_{1,151} \\
\vdots & \vdots & \ddots & \vdots \\
c_{113,1} & c_{113,2} & \ldots & c_{113,151}
\end{array}\right)
$$

where $c_{i, j}$ is one if the grid point is within the center and zero if not.

The center contrail frequency index is defined as the number of aircraft flying through contrail area at time $t$ at level $l$, formulated as

$$
C F I_{\text {center }, l, t}=\sum_{i=1}^{113} \sum_{j=1}^{151} r_{i, j} a_{i, j} c_{i, j}
$$

where $r_{i, j}, a_{i, j}, c_{i, j}$ are defined in Eqn. (2), (3), (4).

The contrail frequency index derived in the previous section indicates the actual contrail activities. For strategic planning, prediction of the contrail frequency at time $t$ at level $l$ of a certain center is calculated by

$$
C F I_{\text {center }, l, t}=\sum_{i=1}^{113} \sum_{j=1}^{151} r_{i, j}^{\prime} a_{i, j}^{\prime} c_{i, j}
$$

where $r_{i, j}^{\prime}$ is from RUC forecast data and $a_{i, j}^{\prime}$ is the predicted aircraft locations from historical air traffic data.

\section{Problem Formulation and Contrail Reduction Strategy}

The center contrail frequency index can be used to identify the flight level that would have formed the most contrails and find an alternate cruising altitude with less contrail formations. The contrail frequency index after the contrail reduction strategy has been applied is formulated as

$$
C F I_{\text {center }, l, t}=\sum_{i=1}^{113} \sum_{j=1}^{151} r_{i, j} c_{i, j} \hat{a}_{i, j}
$$

where $r_{i, j}$ and $c_{i, j}$ are defined in Eqn. (2) and (4) and $\hat{a}_{i, j}$ is defined in Eqn. (3) with the aircraft location after applying the contrail reduction strategy, which can be considered as variables. Thus the contrail reduction strategy is to solve the optimization problem, whose objective is to minimize $C F I$ under several constraints. In this paper, the authors consider the fuel efficiency constraint and the operationally feasible constraints.

\section{A. Fuel Efficiency Constraint}

As discussed in ${ }^{12}$ the original cruising altitude filed in flight plan is usually the optimal cruising altitude in term of fuel efficienccy and aircraft performance. Therefore shifting aircraft up and down to other further levels is more fuel consuming than flying them at original altitude. In this paper, we allow aircraft to alternate the cruise level within the range (block altitude clearance ${ }^{19}$ ) $\left[l-\Delta l_{f e}, l+\Delta l_{f e}\right]$, where $l$ is the 
original cruising level and $\Delta l_{f e}$ is the level changing constraint based on fuel efficiency. $\Delta l_{f e}$ is set to 1 and 2 respectively in our simulation.

As an example, the contrail freqency index matrix at Atlanta Center at 8AM EDT on August 1, 2007 is

$$
C F I_{\text {center }, t}=\left(\begin{array}{ccccccccccc}
\mathbf{0} & 0 & 0 & 0 & 0 & 0 & 0 & 0 & 0 & 0 & 0 \\
0 & \mathbf{0} & 0 & 0 & 0 & 0 & 0 & 0 & 0 & 0 & 0 \\
0 & 0 & \mathbf{0} & 0 & 0 & 0 & 0 & 0 & 0 & 0 & 0 \\
0 & 1 & 0 & \mathbf{0} & 0 & 0 & 2 & 0 & 0 & 1 & 0 \\
28 & 26 & 31 & 33 & \mathbf{1 0} & 52 & 68 & 105 & 51 & 6 & 7 \\
57 & 74 & 43 & 25 & 13 & \mathbf{7 6} & 104 & 148 & 71 & 5 & 10 \\
69 & 29 & 44 & 33 & 28 & 105 & \mathbf{1 2 8} & 209 & 132 & 9 & 14 \\
16 & 12 & 5 & 8 & 5 & 62 & 47 & \mathbf{3 6} & 22 & 6 & 0 \\
0 & 2 & 0 & 0 & 5 & 65 & 45 & 35 & \mathbf{1 9} & 6 & 0 \\
0 & 0 & 0 & 0 & 4 & 71 & 108 & 36 & 19 & \mathbf{6} & 0 \\
0 & 0 & 0 & 0 & 10 & 74 & 122 & 33 & 19 & 0 & \mathbf{0}
\end{array}\right),
$$

where the bold diagonal item $C F I_{\text {center,t, } l}^{l}$ is the contrail freqency index at level $l$ before applying contrail reduction and the off diagonal item $C F I_{\text {center,t, } l}^{l^{\prime}}$ at $\left(l^{\prime}, l\right)$ is the contrail freqency index when guiding aircraft from level $l$ to level $l^{\prime}$. There are 11 levels in total as illustrated in Table 1, therefore the matrix (8) is $11 \times 11$.

If the aircraft are only allowed to move two levels up or down $\left(\Delta l_{f e}=2\right)$ the matrix becomes

$$
\left(\begin{array}{ccccccccccc}
\mathbf{0} & 0 & 0 & \times & \times & \times & \times & \times & \times & \times & \times \\
0 & \mathbf{0} & 0 & 0 & \times & \times & \times & \times & \times & \times & \times \\
0 & 0 & \mathbf{0} & 0 & 0 & \times & \times & \times & \times & \times & \times \\
\times & 1 & 0 & \mathbf{0} & 0 & 0 & \times & \times & \times & \times & \times \\
\times & \times & 31 & 33 & \mathbf{1 0} & 52 & 68 & \times & \times & \times & \times \\
\times & \times & \times & 25 & 13 & \mathbf{7 6} & 104 & 148 & \times & \times & \times \\
\times & \times & \times & \times & 28 & 105 & \mathbf{1 2 8} & 209 & 132 & \times & \times \\
\times & \times & \times & \times & \times & 62 & 47 & \mathbf{3 6} & 22 & 6 & \times \\
\times & \times & \times & \times & \times & \times & 45 & 35 & \mathbf{1 9} & 6 & 0 \\
\times & \times & \times & \times & \times & \times & \times & 36 & 19 & \mathbf{6} & 0 \\
\times & \times & \times & \times & \times & \times & \times & \times & 19 & 0 & \mathbf{0}
\end{array}\right)
$$

where $x$ indicates an invalid move. More strictly, if the aircraft can only be shifted up or down one level $\left(\Delta l_{f e}=1\right)$ the matrix is

$$
\left(\begin{array}{ccccccccccc}
\mathbf{0} & 0 & \times & \times & \times & \times & \times & \times & \times & \times & \times \\
0 & \mathbf{0} & 0 & \times & \times & \times & \times & \times & \times & \times & \times \\
\times & 0 & \mathbf{0} & 0 & \times & \times & \times & \times & \times & \times & \times \\
\times & \times & 0 & \mathbf{0} & 0 & \times & \times & \times & \times & \times & \times \\
\times & \times & \times & 33 & \mathbf{1 0} & 52 & \times & \times & \times & \times & \times \\
\times & \times & \times & \times & 13 & \mathbf{7 6} & 104 & \times & \times & \times & \times \\
\times & \times & \times & \times & \times & 105 & \mathbf{1 2 8} & 209 & \times & \times & \times \\
\times & \times & \times & \times & \times & \times & 47 & \mathbf{3 6} & 22 & \times & \times \\
\times & \times & \times & \times & \times & \times & \times & 35 & \mathbf{1 9} & 6 & \times \\
\times & \times & \times & \times & \times & \times & \times & \times & 19 & \mathbf{6} & 0 \\
\times & \times & \times & \times & \times & \times & \times & \times & \times & 0 & \mathbf{0}
\end{array}\right) .
$$

The following two constraints are called operationally feasible constraints for contrail reduction. 


\section{B. Center Level Capacity Constraint}

In, ${ }^{12}$ the authors shifted all the aircraft at level $l$ to the level with minimum $C F I_{\text {center, }, l, l}^{l^{\prime}}$. However, if there is one level always with the minimum $C F I_{\text {center }, t, l}^{l^{\prime}}$, the previous approach may potentially form a congested center level by moving a large number of aircraft to the same level. In practice, the wake turbulence or wake vortex phenomenon ${ }^{20,21}$ constrains the air traffic controller to maintain a minimum miles-in-trail. The waketurbulence separation criterion is currently a limiting factor in airspace capacities. The FAA is working with NASA to develop and demonstrate integrated systems technology for addressing separation criteria. Some work has been done on miles-in-trail separation and space metering. ${ }^{22,23,24}$ In summary, it is potentially harmful to move too many aircraft to the same level because of smaller separations and associated higher risk. In Fig. 3, the miles-in-trail (MIT) changes from MIT1 to a smaller value MIT3 after the metering point because there are some aircraft merging from the lower level.

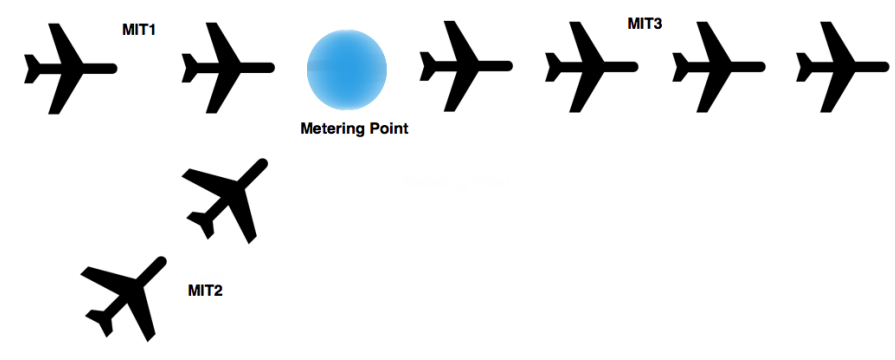

Figure 3. Miles-in-trail is reduced after more aircraft merge from the lower level.

Another reason to introduce this constraint is to keep the air traffic controller's workload below a reasonable threshold. In order to measure dynamic density and evaluate the controller's workload, several metrics were proposed. ${ }^{25,26}$ Among all these dynamic density metrics, the aircraft count or the traffic density in one area is the first factor noticed by every metric. Therefore maintaining the aircraft count at each level below a threshold value will guarantee the workload of air traffic controller manageable.

In this paper, we propose the center level capacity constraint to keep the aircraft amount at each level lower than a critical value. This idea is similar to the Monitor Alert Parameter of a sector, which is set to reflect controller's acceptable workload. ${ }^{27}$ We use our level capacity constraint as a straightforward and operationally intuitive method to keep the controller workload at a manageable level.

\section{Climbing/Descending Aircraft Count Constraint}

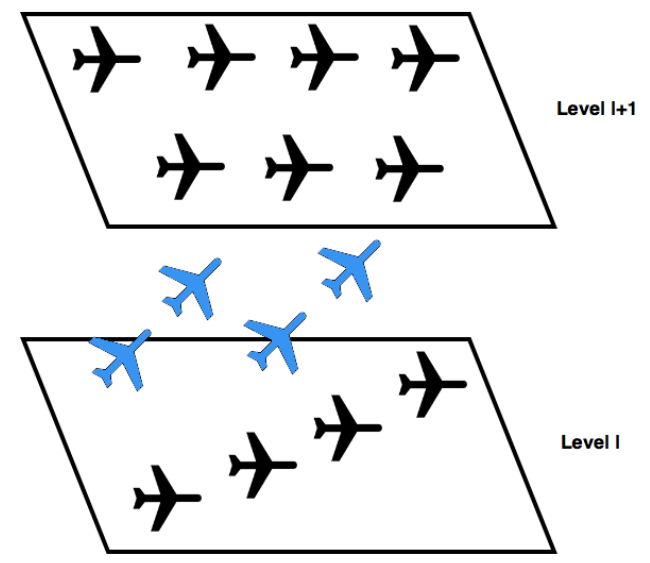

Figure 4. Aircraft are climbing from cruising level $l$ to level $l+1$.

Normally it is difficult for the controllers to visualize potential vertical conflicts with a 2-D radar scope. ${ }^{19}$ 
As shown in Fig. 4, controllers can observe and manage the horizontal aircraft (in black) at level $l$ and level $l+1$ respectively. However, if there are too many climbing aircraft (in blue) moving from level $l$ to level $l+1$, it will introduce extra workload to air traffic controllers.

Almost all the metrics in ${ }^{25,26}$ mentioned the number of climbing/descending aircraft or the number of aircraft with large altitude change as the dynamic density impact factor, which means a huge number of climbing/descending will increase the controllers' workload.

In this study, suppose the solution of aircraft count at level $l$ at time $t$ is $q_{c e n t e r, t, l}^{*}$, we restrict the differences of current solution with the aircraft counts at time $t-1$ and $t+1$ within a given threshold. i.e., $\left|q_{\text {center, }, l, l}^{*}-q_{\text {center }, t-1, l}\right| \leq \Delta q$ and $\left|q_{\text {center }, t, l}^{*}-q_{\text {center }, t+1, l}\right| \leq \Delta q$. By doing this, our contrail reduction strategy should not shift too many aircraft up and down compared to the original flight plans. Therefore less extra workload will be added to the controllers.

\section{Problem Formulation}

Instead of choosing the aircraft count in each grid as the variables, we use the aggregate aircraft count at each level to take advantage of the special structure of contrail frequency index matrix in Eqn. (8).

For ease of illustration, $\Delta l_{f e}$ is set to 1 at this time. Thus our variables are chosen based on the $+/-1$ level changing scheme in Eqn. (10), and the unknown variables are arranged as a vector of length 31

$$
\left[x_{1,1}, x_{2,1}, x_{1,2}, x_{2,2}, x_{3,2}, \ldots, x_{9,10}, x_{10,10}, x_{11,10}, x_{10,11}, x_{11,11}\right]^{\prime},
$$

where $x_{i, j}$ is the number of aircraft which used to cruise at level $j$ and now cruise at level $i$.

Our goal is to guide aircraft to the level with minimum $C F I$ value while satisfying all the constraints. According to Eqn. (10), the $c$ vector in the objective function is:

$$
c=[0,0,0,0,0,0,0,0,0,0,33,0,10,13,52,76,105,104,128,47,209,36,35,22,19,19,6,6,0,0,0]^{\prime} .
$$

Fuel Efficiency Constraint tells us the aircraft used to cruise at level $j$ can only be adjusted to level $j-1, j$ and $j+1$ (except the aircraft used to cruise at the lowest level 1 and the highest level 11). By flow conservation, we have matrix $A_{1}$ of $11 \times 31$ and vector $b_{1}$ of length 11 :

$$
\begin{gathered}
A_{1}=\left(\begin{array}{ccccccccccc}
1 & 1 & 0 & 0 & 0 & \ldots & 0 & 0 & 0 & 0 & 0 \\
0 & 0 & 1 & 1 & 1 & \ldots & 0 & 0 & 0 & 0 & 0 \\
\vdots & \vdots & \vdots & \vdots & \vdots & \vdots & \vdots & \vdots & \vdots & \vdots & \vdots \\
0 & 0 & 0 & 0 & 0 & \ldots & 1 & 1 & 1 & 0 & 0 \\
0 & 0 & 0 & 0 & 0 & \ldots & 0 & 0 & 0 & 1 & 1
\end{array}\right), \\
b_{1}=
\end{gathered}
$$

where $b_{1}(l)$ is the number of aircraft which used to cruise at level $l$ in the original flight plans.

Center Level Capacity Constraint requires the total aircraft number at each level after contrail reduction is fewer than the capacity. Since center level capacity is not an implemented setting in practice, we estimated the values of the capacities for each level. For example, there can be three source streams of aircraft forming the aircraft cruising at level $l$, which are $x_{l, l-1}$ aircraft from level $l-1, x_{l, l}$ aircraft staying at level $l$ and $x_{l, l+1}$ aircraft shifted from level $l+1$. In summary, we obtain constraint matrix $A_{2}$ of $11 \times 31$ and vector $b_{2}$ of length 11:

$$
\begin{aligned}
A_{2}=\left(\begin{array}{ccccccccccccc}
1 & 0 & 1 & 0 & 0 & 0 & \ldots & 0 & 0 & 0 & 0 & 0 & 0 \\
0 & 1 & 0 & 1 & 0 & 1 & \ldots & 0 & 0 & 0 & 0 & 0 & 0 \\
\vdots & \vdots & \vdots & \vdots & \vdots & \vdots & \vdots & \vdots & \vdots & \vdots & \vdots & \vdots & \vdots \\
0 & 0 & 0 & 0 & 0 & 0 & \ldots & 1 & 0 & 1 & 0 & 1 & 0 \\
0 & 0 & 0 & 0 & 0 & 0 & \ldots & 0 & 0 & 0 & 1 & 0 & 1
\end{array}\right), \\
b_{2}=[15,15,20,25,50,250,250,150,70,30,15]^{\prime},
\end{aligned}
$$

where $b_{2}(l)$ is the center level capacity at level $l$. 
Climbing/Descending Aircraft Count Constraint requires the absolute difference between the adjusted aircraft at level $l$ and the aircraft used to cruise at level $l$ at time $t-1$ smaller than a given threshold. i.e., $\left|q_{\text {center }, t, l}^{*}-q_{\text {center }, t-1, l}\right| \leq \Delta q$. Similarly, the absolute difference between $q_{c e n t e r, t, l}^{*}$ and $q_{c e n t e r, t+1, l}$ should also be less or equal than $\Delta q$.

It is easy to calculate the number of aircraft used to cruise at level $l$ at time $t-1$ and $t+1$ from ASDI aircraft data. However, $\Delta q$ value is not necessarily the same under different altitudes, center or sector boundary shapes and geographic conditions. In the simulation part of this paper we assume all the $\Delta q$ are the same.

Ignoring the center subscript notion, we denote the number of aircraft used to cruise at $t-1$ and $t+1$ at level $l$ as $q_{t-1, l}$ and $q_{t+1, l}$, which we can compute from the historical data. At each level, we restrict the maximal number of climbing/descending aircraft with $\Delta q_{l}$. Then we have:

$$
\begin{aligned}
& -\Delta q_{1} \leq x_{1,1}+x_{1,2}-q_{t+1,1} \leq \Delta q_{1}, \\
& -\Delta q_{2} \leq x_{2,1}+x_{2,2}+x_{2,3}-q_{t+1,2} \leq \Delta q_{2}, \\
& -\Delta q_{11} \leq x_{11,10}+x_{11,11}-q_{t+1,11} \leq \Delta q_{11}, \\
& -\Delta q_{1} \leq x_{1,1}+x_{1,2}-q_{t-1,1} \leq \Delta q_{1}, \\
& -\Delta q_{2} \leq x_{2,1}+x_{2,2}+x_{2,3}-q_{t-1,2} \leq \Delta q_{2}, \\
& -\Delta q_{11} \leq x_{11,10}+x_{11,11}-q_{t-1,11} \leq \Delta q_{11} .
\end{aligned}
$$

Let's denote $A_{2}$ as $A_{t+1,+},\left(q_{t+1}+\Delta q\right)$ as $b_{t+1,+},-A_{2}$ as $A_{t+1,-},\left(-q_{t+1}+\Delta q\right)$ as $b_{t+1,-}, A_{2}$ as $A_{t-1,+}$, $\left(q_{t-1}+\Delta q\right)$ as $b_{t-1,+},-A_{2}$ as $A_{t-1,-},\left(-q_{t+1}+\Delta q\right)$ as $b_{t-1,-}$, the Climbing/Descending Aircraft Count Constraint can be written as:

$$
\left(\begin{array}{c}
A_{t+1,+} \\
A_{t+1,-} \\
A_{t-1,+} \\
A_{t-1,-}
\end{array}\right) x \leq\left(\begin{array}{c}
b_{t+1,+} \\
b_{t+1,-} \\
b_{t-1,+} \\
b_{t-1,-}
\end{array}\right) .
$$

By combining (11)(12)(13), we have the fule efficiency constraint (one equality) and two operationally feasible constraints (five inequalities in total) listed all as inequalities below:

$$
\left(\begin{array}{c}
A_{1} \\
-A_{1} \\
A_{2} \\
A_{t+1,+} \\
A_{t+1,-} \\
A_{t-1,+} \\
A_{t-1,-}
\end{array}\right) x \leq\left(\begin{array}{c}
b_{1} \\
-b_{1} \\
b_{2} \\
b_{t+1,+} \\
b_{t+1,-} \\
b_{t-1,+} \\
b_{t-1,-}
\end{array}\right),
$$

which is simply denoted as:

$$
A x \leq b .
$$

Finally, we formulate an integer programming problem:

$$
\begin{array}{ll} 
& \min c^{\prime} x \\
\text { s.t. } & A x \leq b, \\
& x \geq 0, \text { and } x \in \mathbb{I} .
\end{array}
$$




\section{E. Solution - Refined Contrail Reduction Strategy}

In this work the integer programming (15) is relaxed to a linear programming (16) for computational efficiency.

$$
\begin{array}{ll} 
& \min c^{\prime} x \\
\text { s.t. } & A x \leq b, \\
& x \geq 0
\end{array}
$$

We study the total unimodularity of matrix $A$ in (16) and prove that there exists an optimal and integral solution for the linear programming relaxation, which is also the optimal solution for the original integer programming (15). It is guaranteed to be optimal and integral when (16) is solved by the simplex method.

Theorem 1 Matrix A in (16) is total unimodular.

Proof 1 Here the dimension of matrix $A$ is $66 \times 31$. Targeting to transform each row of submatrix $A_{2}$ (14) into the row with a single 1 , through a series of elementary column operations, ${ }^{28}$ we have transformed $A$ as:

$$
\left(\begin{array}{ccccccccccc}
1 & 1 & -1 & -1 & 0 & -1 & 0 & 0 & \ldots & 0 & 0 \\
0 & 0 & 1 & 1 & 1 & 0 & -1 & 0 & \ldots & 0 & 0 \\
0 & 0 & 0 & 0 & 0 & 1 & 1 & 1 & \ldots & 0 & 0 \\
\vdots & \vdots & \vdots & \vdots & \vdots & \vdots & \vdots & \vdots & \vdots & \vdots & \vdots \\
0 & 0 & 0 & 0 & 0 & 0 & 0 & 0 & \ldots & -1 & 0 \\
0 & 0 & 0 & 0 & 0 & 0 & 0 & 0 & \ldots & 0 & -1 \\
0 & 0 & 0 & 0 & 0 & 0 & 0 & 0 & \ldots & 1 & 1 \\
\hline-1 & -1 & 1 & 1 & 0 & 1 & 0 & 0 & \ldots & 0 & 0 \\
0 & 0 & -1 & -1 & -1 & 0 & 1 & 0 & \ldots & 0 & 0 \\
0 & 0 & 0 & 0 & 0 & -1 & -1 & -1 & \ldots & 0 & 0 \\
\vdots & \vdots & \vdots & \vdots & \vdots & \vdots & \vdots & \vdots & \vdots & \vdots & \vdots \\
0 & 0 & 0 & 0 & 0 & 0 & 0 & 0 & \ldots & 1 & 0 \\
0 & 0 & 0 & 0 & 0 & 0 & 0 & 0 & \ldots & 0 & 1 \\
0 & 0 & 0 & 0 & 0 & 0 & 0 & 0 & \ldots & -1 & -1 \\
\hline 1 & 0 & 0 & 0 & 0 & 0 & 0 & 0 & \ldots & 0 & 0 \\
0 & 1 & 0 & 0 & 0 & 0 & 0 & 0 & \ldots & 0 & 0 \\
0 & 0 & 0 & 0 & 1 & 0 & 0 & 0 & \ldots & 0 & 0 \\
\vdots & \vdots & \vdots & \vdots & \vdots & \vdots & \vdots & \vdots & \vdots & \vdots & \vdots \\
0 & 0 & 0 & 0 & 0 & 0 & 0 & 0 & \ldots & 1 & 0 \\
\hline \vdots & \vdots & \vdots & \vdots & \vdots & \vdots & \vdots & \vdots & \vdots & \vdots & \vdots
\end{array}\right)
$$

where the part between the second and the third horizontal lines is the transformed submatrix $A_{2}$. Since matrices $A_{t+1,+}, A_{t+1,-}, A_{t-1,+}, A_{t-1,-}$ in (14) are either $A_{2}$ or $-A_{2}$, every line in the omitted part below the third horizontal line also only contains one single 1 or one single -1 .

Based on (43)(v) of, 29 if the transformed submatrices $A_{1}$ and $-A_{1}$ above the second horizontal line in (17) is totally unimodular, then the original matrix $A$ is also totally unimodular. Moreover, if the transformed $A_{1}$ is total unimodular, then the two parts of the transformed $A_{1}$ and $-A_{1}$ above the second horizontal line are total unimodular. 
Now let's prove the following matrix is total unimodular:

$$
\left(\begin{array}{ccccccccccc}
1 & 1 & -1 & -1 & 0 & -1 & 0 & 0 & \ldots & 0 & 0 \\
0 & 0 & 1 & 1 & 1 & 0 & -1 & 0 & \ldots & 0 & 0 \\
0 & 0 & 0 & 0 & 0 & 1 & 1 & 1 & \ldots & 0 & 0 \\
\vdots & \vdots & \vdots & \vdots & \vdots & \vdots & \vdots & \vdots & \vdots & \vdots & \vdots \\
0 & 0 & 0 & 0 & 0 & 0 & 0 & 0 & \ldots & -1 & 0 \\
0 & 0 & 0 & 0 & 0 & 0 & 0 & 0 & \ldots & 0 & -1 \\
0 & 0 & 0 & 0 & 0 & 0 & 0 & 0 & \ldots & 1 & 1
\end{array}\right)
$$

where each column either contains only one number 1 or a 1/-1 pair. By (43)(v) of, ${ }^{29}$ the first two columns with one non-zero item 1 can be removed while preserving the total unimodularity of a matrix. Then according to (18) of, ${ }^{29}$ the matrix whose each column contains exactly one 1 and exactly one 1 is totally unimodular.

Since matrix $A$ is totally unimodular and vector $b$ is integral, there must exist an optimal and integral solution for both the linear programming relaxation (16) and for the integer programming in (15), which is guaranteed by any simplex method.

In this paper we are dealing with a medium size linear programming problem, so the simplex method in Matlab is capable to provide the efficient solution. The detailed simulation results are presented in next section.

\section{Simulation Results}

In this section, we use the same RUC weather data and ASDI aircraft data as in (8), (9), (10). We first evaluate the performance of our refined strategy after introducing addtional operationally feasible constraints. Secondly, the aircraft count at each cruising level is examined to see if the result of our refined strategy can be governed by the estimated level capacity. Thirdly the numbers of aircraft shifted to each level are plotted which can also be understood as climbing/descending aircraft counts.

\section{A. Contrail Reduction Performance}

In Fig. 5, the indice 1, 2, 3 on $x$-axis represent the historical data (no contrail reduction), the original contrail reduction strategy in ${ }^{12}$ and our refined strategy. The red curve is the result of contrail reduction by allowing $+/-1$ level aircraft shifting. The blue curve is the contrail reduction by allowing $+/-2$ levels aircraft shifting.

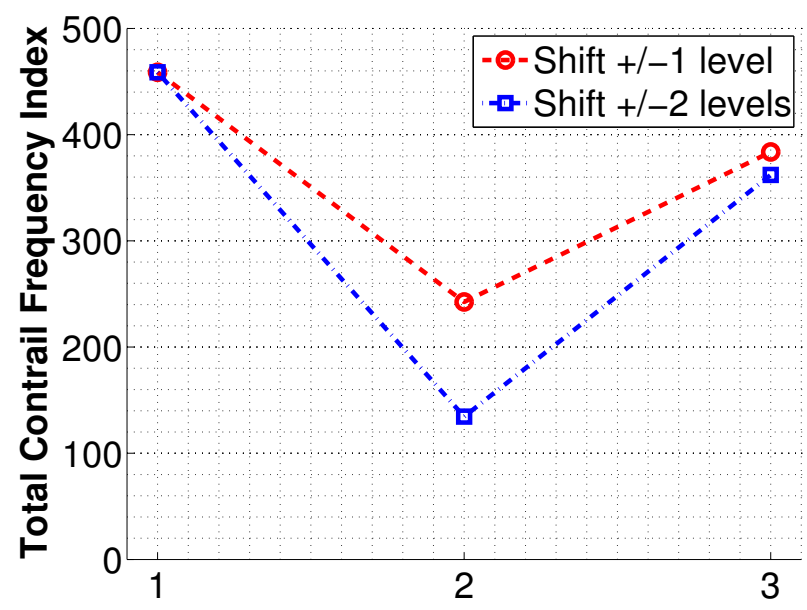

Figure 5. Contrail reduction performance comparison. 
The original strategy has better contrail reduction performance whenever it is the $+/-1$ level shifting or the $+/-2$ levels shifting. The $+/-2$ levels shifting of the original strategy gives out almost $60 \%$ contrail reduction while the $+/-2$ levels shifting of our strategy can provide a $20 \%$ contrail reduction.

\section{B. Level Capacity Constraint}

In Fig. 6 , the numbers $1,2, \ldots, 11$ on $x$-axis are the 11 cruising levels and the $y$-axis indicates the number of aircraft cruising at each level. The red curve describes the level capacity which helps the controllers keep their workload manageable. The blue curve is the adjusted aircraft count at each level provided by the original strategy. The purple curve is the contrail reduction result provided by our refined strategy. The left subfigure is the result of the $+/-1$ level shifting while the right subfigure is the result of the $+/-2$ levels shifting.
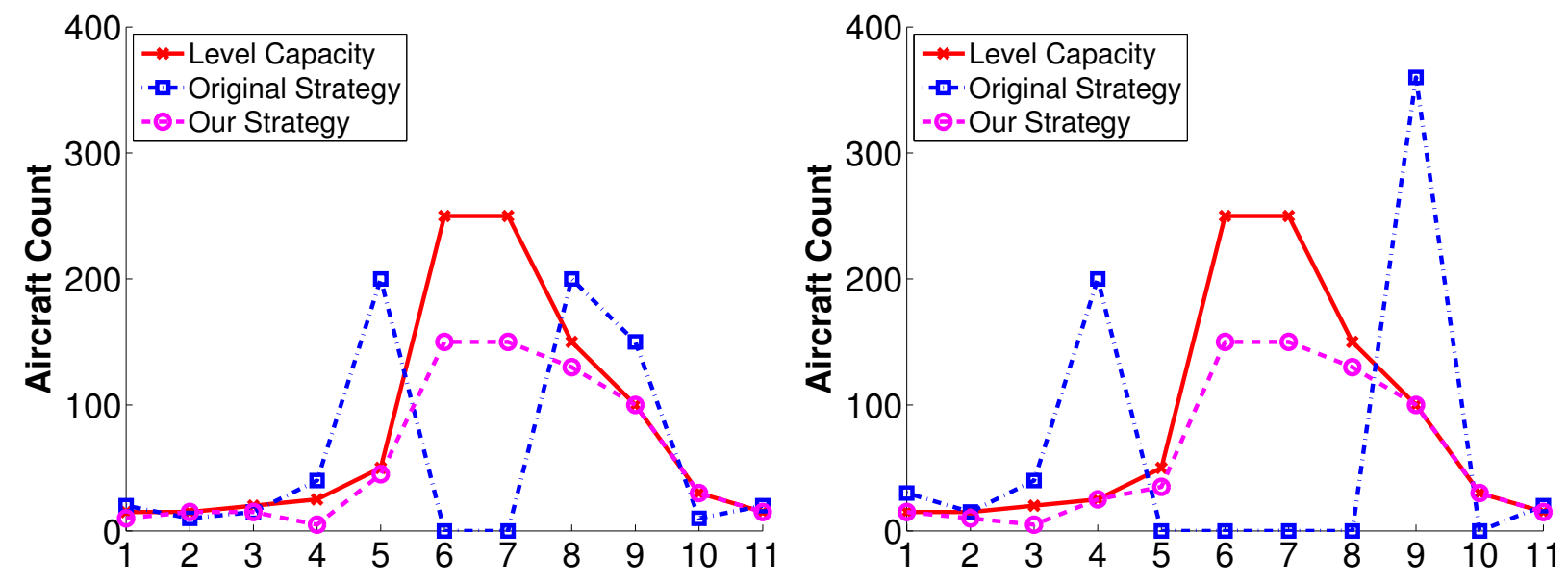

Figure 6. Our refined strategy is strictly governed by the level capacities.

The contrail reduction result from the original strategy will potentially guide the aircraft to the level with minimal contrail potential grids, which may form a very busy cruising level, i.e., level 9 in the right subfigure of Fig. 6. However, our strategy successfully adjust the aircraft to reduce the contrail frequency index and restrict the aircraft count below the level capacity at the same time.

\section{Shifted Aircraft Number}
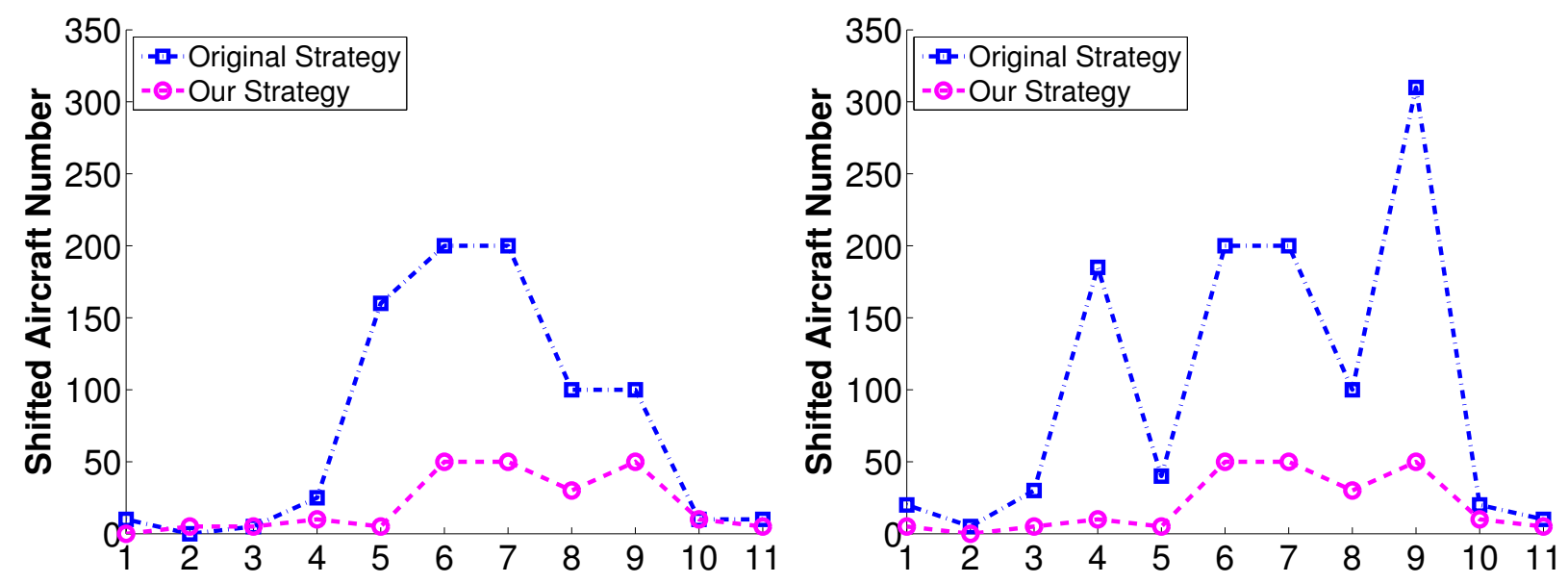

Figure 7. The climbing/descending aircraft count at each level.

Fig. 7 shows us the shifted aircraft number at each level, which can also be understood as climbing/descending aircraft counts. The blue curve represent the result of the original strategy and the purple 
curve is provided by our refined strategy. The left subfigure is the result of the $+/-1$ level shifting while the right subfigure is the result of the $+/-2$ levels shifting.

No matter it is the $+/-1$ level shifting or the $+/-2$ levels shifting, our strategy does great job on managing the aircraft count of each level at a relative low value. This feature can help the controllers process fewer climbing/descending aircraft at each level.

\section{Conclusion}

This study brings two additional operationally feasible constraints to the original contrail reduction problem. By introducing the center level capacity constraint and the climbing/descending aircraft count constraint, the paper formulates the $3 \mathrm{D}$ grid contrail reduction problem as an integer programming. With the total unimodular property, the relaxed linear programming provides the optimal and integral solution in a computational efficient manner, which is the most important advantage of our approach. Another benefit of the method is that instead of shifting all the aircraft from one level to another, our formulation supports the feature of splitting the total number of aircraft used to cruise at level $l$ to multiple levels. i.e., part of the aircraft used to cruise at level $l$ can stay at this level, part of them may be shifted to upper levels, and the remaining part will be guided to lower allowed cruising levels. Simulation results show that the refined contrail reduction strategy provides less contrail reduction than the original strategy presented in. ${ }^{12}$ However, it strictly obeys the level capacity and the numbers of shifted aircraft at each level are restricted to maintain air traffic controllers workload. Future work will integrate operationally acceptable rerouting technique $^{30,31}$ into contrail avoidance trajectory optimization. Instead of the 3D grid aircraft shifting, the new avoidance trajectories and flight plans may provide more contrail reductions.

\section{Acknowledgements}

The authors would like to thank Dr. Craig Wanke from MITRE for the very helpful discussion on operational feasibility constraints and Mr. Dave Slosson, who worked as a FAA air traffic controller at ZID center and then an air traffic supervisor at Fort Wayne tower and TRACON, for his expert and experienced consultation.

\section{References}

\footnotetext{
${ }^{1}$ Meerkotter, R., Schumann, U., Doelling, D., Minnis, P., Nakajima, T., and Tsushima, Y., "Radiative forcing by contrails," Annales Geophysicae, Vol. 17, 1999, pp. 1080-1094.

${ }^{2}$ Marquart, S., Ponater, M., Mager, F., and Sausen, R., "Future Development of Contrail Cover, Optical Depth and Radiative Forcing: Impacts of Increasing Air Traffic and Climate Change," Journal of Climate, Vol. 16, September 2003, pp. 2890-2904.

3 "The Environmental Effects of Civil Aircraft in Flight," Tech. rep., Royal Commission on Environmental Pollution, London, UK, 2002.

${ }^{4}$ Mannstein, H. and Schumann, U., "Aircraft induced contrail cirrus over Europe," Meteorologische Zeitschrift, Vol. 14, No. 4, 2005, pp. 549-554.

${ }^{5}$ Gierens, K., Limb, L., and Eleftheratos, K., "A review of various strategies for contrail avoidance," The Open Atmospheric Science Journal, Vol. 2, 2008, pp. 1-7.

${ }^{6}$ Noppel, F. and Singh, R., "Overview on contrail and cirrus cloud avoidance technology," Journal of Aircraft, Vol. 44, No. 5, 2007, pp. 1721-1726.

${ }^{7}$ Mannstein, H., Spichtinger, P., and Gierens, K., "A note on how to avoid contrail cirrus," Transportation Research. Part D, Transport and environment, Vol. 10, No. 5, September 2005, pp. 421-426.

${ }^{8}$ Campbell, S. E., Neogi, N. A., and Bragg, M. B., "An optimal strategy for persistent contrail avoidance," AIAA Guidance, Navigation and Control Conference, AIAA, Honolulu, HI, August 2008.

${ }^{9}$ Fichter, C., Marquart, S., Sausen, R., and Lee, D. S., "The impact of cruise altitude on contrails and related radiative forcing," Meteorologische Zeitschrift, Vol. 14, No. 4, August 2005, pp. 563-572.

${ }^{10}$ Williams, V., Noland, R. B., and Toumi, R., "Reducing the climate change impacts of aviation by restricting cruise altitudes," Transportation Research. Part D, Transport and environment, Vol. 7, No. 5, November 2002, pp. 451-464.

${ }^{11}$ Williams, V. and Noland, R. B., "Variability of contrail formation conditions and the implications for policies to reduce the climate impacts of aviation," Transportation Research. Part D, Transport and environment, Vol. 10, No. 4, July 2005, pp. 269-280.

${ }^{12}$ Sridhar, B., Chen, N. Y., and Ng, H. K., "Fuel efficient strategies for reducing contrail formations in United States airspace," AIAA Digital Avionics Systems Conference, AIAA, Salt Lake City, UT, Oct 2010.
} 
${ }^{13}$ Minnis, P., Ayers, J. K., Nordeen, M. L., and Weaver, S. P., "Contrail frequency over the united states from surface observations," Journal of Climate, Vol. 16, No. 21, November 2003.

${ }^{14}$ Palikonda, R., Minnis, P., Duda, D. P., and Mannstein, H., "Contrail coverage derived from 2001 AVHRR data over the continental United States of America and surrounding areas," Meteorologische Zeitschrift, Vol. 14, No. 4, August 2005, pp. 525-536.

${ }^{15}$ Duda, D. P., Palikonda, R., and Minnis, P., "Relating observations of contrail persistence to numerical weather analysis output," Atmospheric Chemistry and Physics, Vol. 9, No. 4, February 2009, pp. 1357-1364.

${ }^{16}$ Duda, D. P., Minnis, P., Costulis, P. K., and Palikonda, R., "Conus contrail frequency estimated from ruc and flight track data," European Conference on Aviation, Atmosphere, and Climate, Friedrichshafen at Lake Constance, Germany, June-July 2003.

${ }^{17}$ Alduchov, O. A. and Eskridge, R. E., "Improved magnus form approximation of saturation vapor pressure," Journal of Applied Meteorology, Vol. 35, No. 4, April 1996, pp. 601-609.

${ }^{18}$ Chen, N. Y., Sridhar, B., and Ng, H. K., "Prediction and use of contrail frequency index for contrail reduction strategies," AIAA Guidance, Navigation and Control Conference, AIAA, Toronto, Canada, Aug 2010.

${ }^{19}$ Nolan, M. S., Fundamentals of Air Traffic Control, Cengage Learning, 5th ed., Feb 2010.

${ }^{20}$ Hay, G. C. and Passman, R. H., "Wake Turbulence Training Aid," Tech. rep., John A. Volpe National Transportation Systems Center, Cambridge, MA, 1995.

${ }^{21}$ Speijker, L., van Baren, G., Sherry, L., Shortle, J., and Rico-Cusi, F., "Assessment of Wake Vortex Separation Distances Using the WAVIR Toolset," AIAA Digital Avionics Systems Conference, San Diego, USA, 2004.

${ }^{22}$ Kopardekar, P., Green, S., Roherty, T., and Aston, J., "Miles-in-trail Operations: A Perspective," AIAA's 3rd Aviation Technology, Integration, and Operations (ATIO) Tech, Denver, USA, November 2003.

${ }^{23}$ Wang, P., Wanke, C., and Wieland, F., "Modeling time and space metering of flights in the National Airspace System," Simulation Conference, Washington, DC, USA, 2004, pp. 1299-1304.

${ }^{24} \mathrm{Ny}$, J. L. and Pappas, G. J., "Joint Metering and Conflict Resolution in Air Traffic Control," Tech. rep., University of Pennsylvania, 2010.

${ }^{25}$ Sridhar, B., Sheth, K. S., and Grabbe, S., "Airspace Complexity and its Application in Air Traffic Management," 2nd USA-Europe ATM Seminar, Orlando, USA, December 1998.

${ }^{26}$ Kopardekar, P. and Magyarits, S., "Measurement And Prediction Of Dynamic Density," 5th USA-Europe ATM Seminar, Budapest, Hungary, June 2003.

${ }^{27}$ Federal Aviation Administration, Facility Operation and Administration, Chapter 17, Section \%. Monitor Alert Parameter, 2008, Order JO 7210.3V.

${ }^{28}$ Wei, P. and Sun, D., "Total Unimodularity and Degeneracy-Aware Dantzig-Wolfe Decomposition for Large Capacity Cell Transmission Model," 50th IEEE Conference on Decision and Control and European Control Conference, Orlando, USA, Dec 2011.

${ }^{29}$ Schrijver, A., Theory of Linear and Integer Programming, Wiley, 1998.

${ }^{30}$ Taylor, C. and Wanke, C., "Dynamic Generation of Operationally Acceptable Reroutes," 9th AIAA Aviation Technology, Integration, and Operations Conference, Hilton Head, South Carolina, Sep 2009.

${ }^{31}$ Taylor, C. and Wanke, C., "Improved Dynamic Generation of Operationally Acceptable Reroutes Using Network Optimization," Journal of Guidance, Control, and Dynamics, 2011. 